\title{
Connecting Envisioning Process to User Interface Design Process
}

\author{
Naotake Hirasawa ${ }^{1}$, Shinya Ogata ${ }^{2}$, and Kiko Yamada-Kawai ${ }^{1}$ \\ ${ }^{1}$ Otaru University of Commerce, Midori 3-5-21, Otaru, Japan 047-8501 \\ \{hirasawa, kawai\} @res . otaru-uc.ac.jp \\ ${ }^{2}$ U'eyes design Inc., Housquare Yokohama 4F, Nakagawa 1-4-1, Tsuzuki-ku, Japan 224-001 \\ ogata@ueyesdesign.co.jp
}

\begin{abstract}
As embedded systems have increasingly become multifunctional, it has become necessary to clarify what sorts of experiences the user can have through these functions. It is the system's user interface (UI) that leads this user experience, therefore, UI design has increased in importance. However, conventional design processes, often set the UI design after defining the system requirements, which causes operations to become more complex and leads to a lack of operational consistency. To overcome these problems, a design process was conceived whereby the user experience envisioned in the product planning phase is realized in the UI. Two workshops were held for development-related personnel to introduce the conceived process into actual development projects. The effectiveness of the process was acknowledged by all the participants, but they also pointed out the difficulty of incorporating the process into their organizations.
\end{abstract}

Keywords: User Interface Design, Design Processes, Workshop.

\section{Introduction}

In developing embedded systems, the importance of the UI design has been increasingly recognized. The means of operating the system functions are realized into the UI in the embedded system. When the functions are few, the UI is simple and easy to operate. A rapid advance in infrastructure technology, however, has enabled the system to incorporate a multitude of functions; consequently, UIs have become complicated. Systems now face the contradiction that convenient functions are incorporated but they cannot be fully utilized as the operation becomes too complicated.

At the same time, the linkage with telecommunications technology has enabled a system to connect to the information infrastructure and other systems, providing it with intermediary roles for various services along with services of its own. The cell phone is a typical example as it started from a single phone unit to evolve into an information terminal that now intermediates various other services. This means a UI needs to be considered at the phase of development where the services to be provided by the products are conceived. In other words, the UI must start to be considered in the product planning phase. 
Taking into consideration these changes in the role of the UI in an embedded system, our research project has created a design process that allows the user experiences (UX) envisioned in the planning phase to be realized into the UI, while balancing the operational system as a whole.

This paper describes a summary of this process model, and discusses the issues involved in the organizational implementation of the process, which were based on the analysis of the results of workshops held for company employees engaged in development.

\section{Process Model}

\subsection{Problems of UI Design in Embedded System Development}

In general, UI design proceeds based on the functional and non-functional requirements that are clarified after the system requirement definition have been conducted. In this process, time is so limited by software design that the UI design is often implemented in the system without performing any validation from the user's perspective. Even when UI design is outsourced, UI specification is often designed under conditions where all the functions to be implemented have already been determined. In such cases, the following problems will arise in designing the UI during the embedded system development [1].

- There is a possibility that the UXs envisioned in the planning process are not necessarily reflected in the UI.

- The direction of the multifunctionality of the system is not controlled, so operational systems become complicated.

- Validation of the UI specification becomes difficult.

- Management in changing the UI specification becomes difficult.

- To design the optimal UI specification, user researches conducted in the planning phase sometimes need to be performed again.

To overcome these problems, such measures are available as (i) coordinating the requirements for defining the embedded system and for designing the UI; and (ii) making the background of the UI specification traceable. Besides these, our project implemented the following two new measures.

1) Introduction of the concept "view for context of use"

The performance of designing UI over the past 20 years at U'eyes Design Inc. clearly shows that the defining view that characterize activities of the users of the system can facilitate UI design [2]. For example, if we consider the UI for displaying the sales performance of a POS system, the UI can be designed from at least three different viewpoints: from the system's goal of checking the sales performance; from the viewpoint of where the system is used; or from the viewpoint of the user characteristics. The important thing is that these viewpoints determine the directions of UX when the system is used. In other words, clarifying the viewpoints will also lead to the identification and clarification of how the system will appeal to the user. 


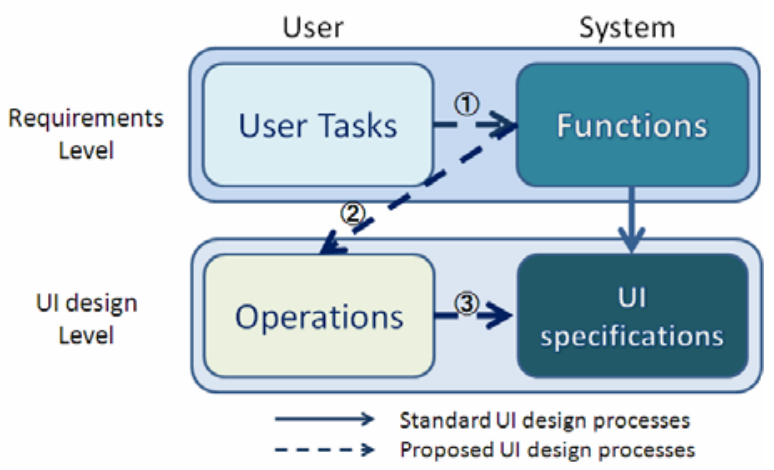

Fig. 1. Depicting a framework of UI design processes

If the concept of view is used, user characteristics will be the viewpoint in the method advocated by Cooper [3]; and it can be regarded that UX is defined from the viewpoint of "work" in Contextual Design [4].

Defining these viewpoints means clarifying the intentions or directions of using the system, and this becomes an aid to determining the direction of the UI during the planning phase. We have named these views as "view for context of use (riyou tokusei in Japanese)" as they determine the nature of the system use.

2) Depicting the concept of proposed UI design processes

The general rule for designing the UI in the embedded system is to design the UI specification based on the functions determined by the definition of the requirements. In this case, the UI design process is people-dependent and the UI is often designed with reference to the conventional UI or a competitor's UI.

We determined the reason for such a practice to be that the basic concepts underlying the UI design process was not shared among members. This was because the background the UI was designed against was ambiguous. To help solve the problem, the following basic concepts were identified regarding the relationship between the UI and the system requirements: (Fig.1)

- The goal of the system functions is to realize user tasks.

- The system functions are used through the user's operation of the system.

- The means to realize the operation of the system is the UI.

If the design process is organized under these concepts, UI design can be positioned as the means to realize user tasks. As a result, UI specification can be traced from the user tasks.

\subsection{Proposed Design Processes Model}

Based on the above-mentioned ideas, it becomes possible to develop a process model that can connect activities from planning to UI design. In developing the process, some development lifecycle models were used: Embedded System development Process Reference (ESPR) developed by Software Engineering Center under Information-Technology Promotion Agency was used as a base [5]; and ISO 15288 
[6] and ISO 12207 [7] were used as reference. Also ISO 13407 [8], ISO 18529 [9], Rapid Contextual Design [4], IBM's UCD model [10], and Cooper's model [3] among others were used, especially for process models during the UI design process (Fig.2).

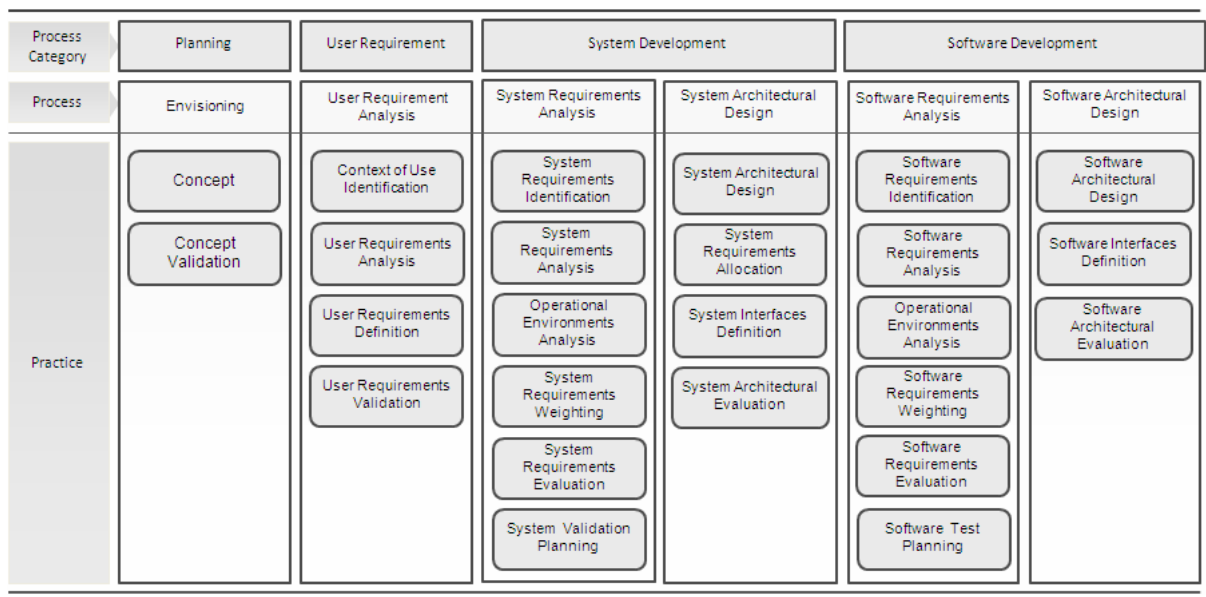

Fig. 2. Outline of HCD Process integration Model

\section{Implementing Workshops Based on Proposed Design Process}

To confirm the validity of the proposed process model, workshops were held for employees engaged in the development in domestic manufacturers, to investigate its possibilities and limitations. Workshops were implemented at the following two companies:

\subsection{Case A (Manufacturing Equipment Maker)}

Background. A large manufacturing equipment maker with over 10,000 employees, this company has created a brand with high quality products and customer-oriented services. The company is aiming to convert its business from providing manufacturing equipment to providing solutions for controlling multiple equipments. In order to fulfill the goal, a new design process needed to be developed. Workshops were held with the expectation that the process model we had conceived would be applied to their new design process.

Participants. 5 engineers of equipment development and software development, and 1 sales-related employee.

Program. A series of four workshops were held from August 2009 to December 2009. Each goal of the workshop was the following;

1 st was to understand new requirements definition processes,

2nd was to identify as-is scenarios for describing contexts of system use, 
3rd was to elicit system requirements,

4th was to consolidate and depict system requirements.

In the program, a solution that had been already developed was used as the object, and various activities were performed from the planning phase through to the system requirements analysis based on the proposed design process. Information necessary for the workshops was searched for and collected before the workshops began. Eventually a definition of the system requirements was established. After all the workshops finished, the participants were asked to evaluate the proposed processes with questionnaire and interviews were conducted for the every participant.

Results. The system requirements analysis was able to clarify the functions that the existing solution lacked. All the participants acknowledged the effectiveness of the proposed design process. Specifically, participants pointed out the possibility of sharing information from the planning phase to the lower level processes. One participant commented that the process provided a bird's-eye view over the upstream processes of the system development, and that therefore, it could be directly applied to educational training.

In terms of the application possibilities of this process to their actual operation, three participants (50\%) said it could be applied. Similarly, three participants said they could immediately apply the process to their own work. Half of the participants pointed out the difficulty of expanding the process to the entire organization. Some expressed anxiety over the possibility of implementing the process by themselves after the workshops were over.

\subsection{Case B (Home Appliance Maker)}

Background. This company is a home appliance maker with a long history and more than 10,000 employees. It has state-of-the-art technologies in some specific technological fields, and has an established brand. However, the market to which the company provides products has matured, and differentiation of products between competitors has become difficult. The company has aimed at differentiation by making the UI of its products more appealing, and it has sought a new UI design process to accomplish this goal. They took this workshop as an opportunity to review their UI design process to develop new products, since UI technology infrastructure was experiencing drastic changes, and they asked us to hold workshops for employees who were engaged in development.

Participants. 2 product planners, 1 product designer, 2 requirements engineers, 2 software engineers, 4 usability engineers (total of 11).

Program. A series of four workshops were held from July 2009 to December 2009. Each goal of the workshop was the following;

1st was to identify the problems of the existing UI design processes,

2nd was to identify the user requirements of a new product,

3rd was to identify the system requirements,

4th was to design the outline of UI specifications. 
Before the workshops, a seminar was prepared to help the participants understand the new design process. The program started by conducting an analysis of the existing design process, then it identified the issues involved in the UI design process, and finally it established the outline specification of the UI of the product under development. Workshops, held in parallel with the product development, were held during the program, adjusted in line with the product development schedule. After the every workshops finished, interviews for the every participant were conducted.

Results. During this program various ideas not obtained from the concurrent design process were obtained. All the participants acknowledged the effectiveness of the proposed design process. Specifically, the program enabled them to give a meaning to the newly derived requirement functions, and to strengthen the product proposal. The applicability of the process to their work was also acknowledged by all. However, more than $30 \%$ of the participants stated that only some individual methods could be applicable, and that the introduction of the process would be difficult. Continual support to usability engineers was mentioned as one important condition for applicability.

Both companies clearly acknowledged the effectiveness of the proposed process. As for the reason for the difference in applicability between the two companies, it can be pointed out that the former company does not offer organized support on humancentered design.

Whether trying to increase the conceptual power for solutions, or trying to enhance the appeal to the product by using UI, it has become clear that there is a big barrier to changing the existing design process, although the effectiveness of the proposed process was acknowledged. One of the issues is how to organize a support system for the proposed process.

\section{Conclusions}

As the embedded system has become increasingly multifunctional, the UI of the system has attracted expectations from the perspective of product appeal, even though it has a number of issues, and the importance of the UI design has increased. However, in the conventional design process, UI design is often conducted in the post-process of defining the system requirements, during the time the operational methods of the required functions are being realized. As a result, many design processes still have such problems as product specification turning out to be filled with functions, and the difficulty of changing the UI specification.

This study proposed a design process that enables the UX envisioned in the product planning phase to be implemented into the system in the form of a UI while balancing the operational system of the entire system. What characterizes this process is the concept of "views for context of use," and the direction given to the process based on the relationship between user tasks and functions.

Workshops were held for development personnel to check the validity of the proposed process model. As a result, the process was acknowledged to be effective, however, participants also pointed out the difficulty of actually introducing the process into their organizations.

Further study is expected on the process maturity model when the process has been introduced into organizations. 
Acknowledgements. The authors would like to thank Toshiyuki Oketani and Hideaki Kasai for coordinating the workshops.

\section{References}

1. Hirasawa, N., Ogata, S., Urokobara, H.: Substantializing User Experience in the Processes for Developing Embedded Systems (in Japanese). Econimic Review 60(4) (2010)

2. Ogata, S., Urokobara, H., Aoshima, H.: Possibility of Modeling for Incorporating UI Design into System Design (in Japanese). In: Proceeding of Human Interface Symposium 2004, pp. 1073-1076 (2004)

3. Cooper, A., Reimann, R., Cronin, D.: About Face 3: The Essentials of Interaction Design. John Wiley \& Sons Inc., Chichester (2007)

4. Beyer, H., Holtzblatt, K., Wood, S.: Rapid Contextual Design: A How-to Guide to Key Techniques for User-Centered Design. Morgan Kaufmann, San Francisco (2004)

5. Software Engineering Center 2007 Developing Process Guide for Embedded Software (in Japanese). Information-technology Promotion Agency, Japan, Syou-Ei-sya (2007)

6. ISO/IEC 15288:2008 Systems and software engineering - System life cycle processes

7. ISO/IEC 12207:2008 Systems and software engineering - Software life cycle processes

8. ISO 13407:1999 Human-centred design processes for interactive systems

9. ISO/TR 18529:2000 Ergonomics - Ergonomics of human-system interaction - Humancentred lifecycle process descriptions

10. Vredenburg, K., Isensee, S., Righi, C.: User-Centered Design: An Integrated Approach. Prentice-Hall, Englewood Cliffs (2001) 